\title{
Accurate molecular dynamics and nuclear quantum effects at low cost by multiple steps in real and imaginary time: using density functional theory to accelerate wavefunction methods
}

\author{
V. Kapil, ${ }^{1}$ J. VandeVondele, ${ }^{2, \sqrt{a})}$ and M. Ceriotti ${ }^{1, b)}$ \\ ${ }^{1)}$ Laboratory of Computational Science and Modelling, Institute of Materials, \\ Ecole Polytechnique Fédérale de Lausanne, Lausanne, Switzerland \\ ${ }^{2)}$ Department of Materials, ETH Zurich, Wolfgang-Pauli-Strasse 27, CH-8093 Zurich, \\ Switzerland
}

(Dated: 2 December 2015)

The development and implementation of increasingly accurate methods for electronic structure calculations mean that, for many atomistic simulation problems, treating light nuclei as classical particles is now one of the most serious approximations. Even though recent developments have significantly reduced the overhead for modeling the quantum nature of the nuclei, the cost is still prohibitive when combined with advanced electronic structure methods. Here we present how multiple time step integrators can be combined with ringpolymer contraction techniques (effectively, multiple time stepping in imaginary time) to reduce virtually to zero the overhead of modelling nuclear quantum effects, while describing inter-atomic forces at high levels of electronic structure theory. This is demonstrated for a combination of MP2 and semi-local DFT applied to the Zundel cation. The approach can be seamlessly combined with other methods to reduce the computational cost of path integral calculations, such as high-order factorizations of the Boltzmann operator, or generalized Langevin equation thermostats.

PACS numbers: 02.70.Ns, 31.15.xk, 31.15.vq

Keywords: nuclear quantum effects, path integrals, density functional theory, wavefunction theory, vibrational spectroscopy, multiple timestep algorithms

Simulations of molecules and materials from first principles are constantly improving in accuracy and predictive power, thanks to the combination of the availability of more powerful high-performance computing (HPC) platforms and the development of more efficient techniques to treat the electronic structure problem at high levels of theory. For example, the structure and dynamics of bulk liquid water can now be computed using correlated wavefunction method $\sqrt{12}$, or quantum Monte Carld $^{3}$. At the same time, these developments pose new challenges. To reach the ultimate level of accuracy it is not sufficient to fully account for exchange and correlations between electrons, but it is also necessary to describe the quantum mechanical nature of light nuclei, such as hydrogen. Modelling of nuclear quantum effects (NQEs) can be achieved by path integral molecular dynamics (PIMD) 46, that traditionally involves a very large overhead. The large computational cost can be traced to the need of simulating multiple replicas of the physical system and to the need for a reduced time step to properly integrate the dynamics of the stiff, non-ergodic modes of the ring polymer Hamiltonian. Indeed, using brute force PIMD techniques, several hundred million core hours would be needed to faithfully model quantum effects in the above mentioned MP2 simulations of liquid water.

\footnotetext{
a) Electronic mail: joost.vandevondele@mat.ethz.ch

b) Electronic mail: michele.ceriotti@epfl.ch
}

However, recent advances have contributed to dramatically reduce this overhead, by using high-order expansions of the finite-temperature density matrix ${ }^{7 / 8}$, by using a correlated-noise Generalized Langevin Equation to induce frequency-dependent fluctuations mimicking quantum effects9-11, or by selectively treating with different number of path integral replicas different parts of the system $\frac{12]}{}$ or different components of the potentia ${ }^{[13}$. In a converged path integral simulation, adjacent replicas in the ring polymer are held close together by the presence of harmonic springs. Whenever it is possible to partition the inter-atomic potential into a rapidly-varying and easily-computed part, and a harder-to-compute but slowly-varying part, one can also reduce the cost of evaluating the path energy by a so-called ring-polymer contraction (RPC) scheme ${ }^{13] 14}$, that only evaluates the expensive component on a reduced number of replicas.

In fact, the idea of exploiting the presence of different length scales in the interactions between atoms has been recognized long since, and has been used to accelerate the integration of classical equations of motion where the granularity of time is set by the stiffest part of the interaction ${ }^{15}$. This intuition can lead to significant speed-ups when the rapidly varying part of the interaction potential can be computed more efficiently than the slowly-varying part - which is e.g. the case for long-range electrostatic interactions ${ }^{16}$, or biasing potentials derived from complex order parameters 17 . The partitioning of interactions in slow (smoothly-varying and expensive to compute) and fast (quickly-varying and inexpensive) components is less obvious when first-principles calculations are considered, 
as individual terms are often hard to treat separately. However, in this context, the vastly different costs between different levels of theory can be exploited. This was first demonstrated in Ref. $\frac{18}{18}$ where a combination of hybrid functionals and semi-local DFT was exploited in a multiple time-step (MTS) scheme. This early work was inspired by the combination of force-fields and DFT for presampling and biasing $\left.{ }^{19} 21\right]$ Different variations on this theme, that all have the potential to be generally applicable, have been shown to be effective. For MTS schemes that have been applied in an $a b$ initio context include the combination of explicitly correlated methods on top of a baseline Hartree-Fock calculation ${ }^{22}$, the application of a range-separated Coulomb operator or a divide-andconquer strategy ${ }^{23}$, the use of better-converged simulation parameters as a correction, considered to be slowly varying,24, or the combination of MP2 and semi-local $\mathrm{DFT}^{2}$. The latter variant will be employed here, yielding a massive speedup by combining MTS and RPC.

\section{COMBINING MULTIPLE TIME STEP INTEGRATORS AND RING-POLYMER CONTRACTION}

MTS and RPC techniques can be combined fairly straightforwardly ${ }^{25}$, and indeed, such a combination was used already in early classical simulations based on ringpolymer contraction 26 . However, to date no attempt has been reported to use RPC (alone or in combination with MTS) in the context of ab initio molecular dynamics, despite the fact that this is a scenario in which obtaining an accurate estimate of nuclear quantum effects at a reduced computational cost would be particularly desirable. This is largely due to the considerable implementation overhead connected with combining sophisticated techniques for solving efficiently the electronicstructure problem and the cumbersome formalism that underlies PIMD and its RPC and MTS extensions. Here we present a simple example that demonstrates a working implementation of such combination, modeling nuclear quantum effects with a post-HF wavefunction description of the electronic structure. Our implementation relies on the modular assembly of i-P127 - a Python interface in which we implemented MTS and RPC techniques - and $\mathrm{CP} 2 \mathrm{~K}^{28}$ - that sports a highly efficient periodic implementation of second-order Møller-Plesset (MP2) theory. Commented example input files, and a snapshot of our development code are provided in the supporting information.

We refer the reader to the relevant literature for the theoretical foundations, and general considerations regarding MTS and RPC techniques 13 15. Here we will just briefly overview the main ideas, using as an example a path integral simulation of a flexible fixed-pointcharge model of water 26 . In the most straightforward formulation, performing a PIMD simulation for a system composed of $N$ distinguishable particles with masses $m_{i}$ and positions $\mathbf{q}_{i}$ amounts to sampling at inverse temper- ature $\beta_{P}=1 / P k_{B} T$ a canonical distribution consistent with the classical Hamiltonian $H_{P}(\mathbf{p}, \mathbf{q})=H_{P}^{0}(\mathbf{p}, \mathbf{q})+$ $V_{P}(\mathbf{p}, \mathbf{q})$, where the free ring-polymer Hamiltonian is

$$
H_{P}^{0}(\mathbf{p}, \mathbf{q})=\sum_{i=1}^{N} \sum_{j=1}^{P}\left(\frac{\left[\mathbf{p}_{i}^{(j)}\right]^{2}}{2 m_{i}}+\frac{1}{2} m_{i} \omega_{P}^{2}\left[\mathbf{q}_{i}^{(j)}-\mathbf{q}_{i}^{(j+1)}\right]^{2}\right)
$$

and the physical potential term is

$$
V_{P}(\mathbf{q})=\sum_{j=1}^{P} V\left(\mathbf{q}_{1}^{(j)}, \ldots, \mathbf{q}_{N}^{(j)}\right)
$$

The ring polymer is composed of $P$ replicas of the physical system (positions $\mathbf{q}_{i}^{(j)}$ ) cyclically connected by springs with frequency $\omega_{P}=P \beta \hbar$. To simplify the discussion of the combination of RPC and MTS, let us split the potential in a slow $(\mathrm{S})$ and a fast $(\mathrm{F})$ part $V_{P}=V_{P^{\prime} \leftrightarrow P}^{\mathrm{S}}+V_{P}^{\mathrm{F}}$. The $V_{P^{\prime} \leftrightarrow P}^{\mathrm{S}}$ notations indicates that the slow part of the potential is evaluated on a "contracted" ring polymer. The $P$-bead path can be Fourier-interpolated down to a smaller number of replicas $\mathrm{P}^{\prime}$, for which energy and forces are computed, and then Fourier-expanded back to $P$ replicas. This makes RPC fully transparent from the point of view of the integration of the equations of motion and the evaluation of system properties, simplifying both notation and implementation. Note that it is often the case that one wants to use a low level of theory for the quickly varying part, $V^{\text {cheap }} \equiv V^{\mathrm{F}}$, and apply on a longer time step or contracted ring polymer a correction term based on a more accurate assessment of the inter-atomic potential $V^{\text {accu }}-V^{\text {cheap }} \equiv V^{\mathrm{S}}$.

As shown in Fig. 1, the convergence of observables to quantum expectation values in a simulation of liquid water at room temperature require a large number of replicas, of the order of $P \approx 30$. However, this is mostly due to the internal modes of each water molecule, that are well described by cheap quasi-harmonic terms. The non-bonded dispersion and electrostatic interactions vary on considerably larger length scales, and therefore a RPC scheme that computes the latter on a reduced number of replicas converges very rapidly. A simple rangeseparation procedure would allow one to reach convergence with a single evaluation of electrostatic interactions 14 . Here we want however just to verify to what extent one can push a MTS procedure, alone or in combination with RPC, in a PIMD context.

A multiple time-step integrator for constanttemperature PIMD is epitomized by the corresponding split operator for the propagator over the outer time step $\Delta t$ :

$$
\begin{aligned}
e^{-\mathcal{L} \Delta t} & =e^{-\left(\mathcal{L}_{\gamma}+\mathcal{L}_{0}+\mathcal{L}_{V}^{\mathrm{F}}+\mathcal{L}_{V}^{\mathrm{S}}\right) \Delta t} \approx e^{-\mathcal{L}_{\gamma} \frac{\Delta t}{2}} e^{-\mathcal{L}_{V}^{\mathrm{S}} \frac{\Delta t}{2}} \times \\
& \times\left[e^{-\mathcal{L}_{V}^{\mathrm{F}} \frac{\Delta t}{2 M}} e^{-\mathcal{L}_{0} \frac{\Delta t}{M}} e^{-\mathcal{L}_{V}^{\mathrm{F}} \frac{\Delta t}{2 M}}\right]^{M} e^{-\mathcal{L}_{V}^{\mathrm{S}} \frac{\Delta t}{2}} e^{-\mathcal{L}_{\gamma} \frac{\Delta t}{2}}
\end{aligned}
$$

First, a thermostat is applied by half the outer time step $\left(\mathcal{L}_{\gamma} \frac{\Delta t}{2}\right)$; then the slowly varying force (possibly computed by RPC) is applied $\left(\mathcal{L}_{V}^{\mathrm{S}} \frac{\Delta t}{2}\right)$; then, the inner loop of 

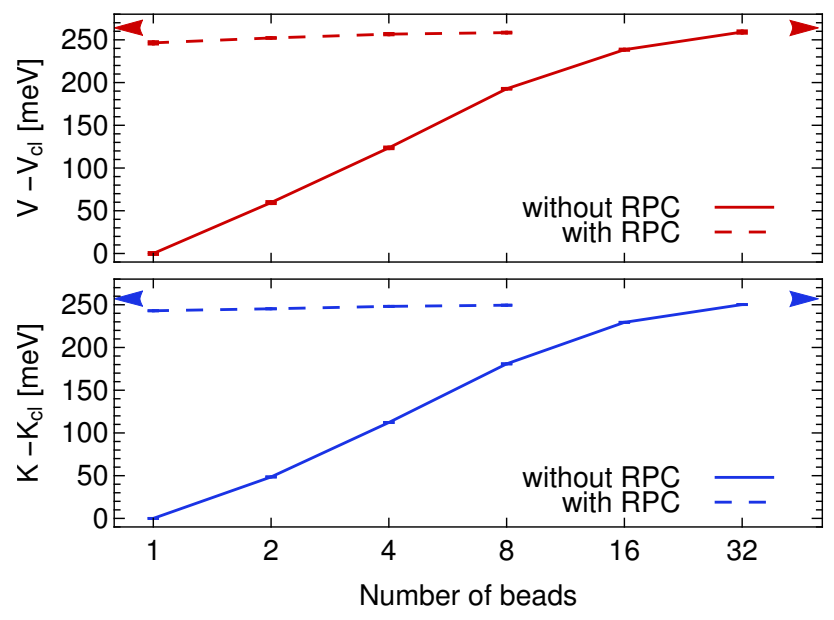

FIG. 1. Difference between quantum and classical expectation values of potential (top) and kinetic energy (bottom) per molecule as a function of the number of path integral replicas for a simulation of $\mathrm{q}-\mathrm{TIP} 4 \mathrm{P} / \mathrm{F}$ liquid water at room temperature. Solid curves correspond to full PIMD, while dashed curves correspond to a RPC setup in which the bonded (stretch and bend) terms were computed on 32 replicas, and the non-bonded (electrostatic and dispersion) terms were computed on a reduced number of replicas, that corresponds to the horizontal axis. The blue and red arrow-heads correspond to full PIMD using 64 beads.

the MTS integrator is performed, repeating $P^{\prime}$ times the combined integration of the fast force component $\left(\mathcal{L}_{V}^{\mathrm{F}}\right)$ and the free-ring-polymer Hamiltonian $\left(\mathcal{L}_{0}\right)$, with a time step duly scaled by a factor of $M$. Finally, the slowly varying force and the thermostat are applied symmetrically, concluding the time step. As discussed e.g. in Refs $\stackrel{29 \mid 30}{20}$, regardless of how well separated the two components of the potential are, it is not possible to extend indefinitely the outer time step, as accumulation of errors will make the integration unstable when $\Delta t$ is larger than a fraction of the highest-frequency vibrational period.

Due to the presence of high-frequency normal modes in the free ring-polymer Hamiltonian (Eq. (1)) one would expect the first occurrence of resonances to appear already at a time step of about $1 \mathrm{fs}$ for a path integral simulation with 32 replicas at $300 \mathrm{~K}^{23}$, much earlier than the $\approx 2.7 \mathrm{fs}$ limit that would be expected due to the stretching mode frequency. Figure 2 shows that in this particular case the PIMD simulation remains stable up to an outer step of about $2 \mathrm{fs}$, even in a weaklythermostatted (white-noise Langevin, thermostat correlation time of $2 \mathrm{ps}$ ) PIMD simulation. This rather puzzling finding can probably be ascribed to the exceedingly weak coupling between the physical potential and the high-frequency normal modes of the ring polymer, and does not necessarily apply to different systems with stronger anharmonicities. Here, increasing the dynamical masses of high-frequency ring-polymer modes would delay only minimally the resonance barrier, and would not be possible when using approximate quantum dynamics techniques, such as centroid molecular dynamics (CMD $)^{31}$ and thermostatted ring polymer molecular dynamics (TRPMD) ${ }^{32133}$, that involve specific prescriptions for the magnitude of the dynamical masses. However, a similarly effective solution to deal with ringpolymer resonance barriers is to selectively thermalize the high-frequency vibrations 34 . Exploiting the approximate knowledge of high-frequency ring polymer modes, one can here simply use optimally-damped Langevin thermostats in the (ring-polymer) normal-modes representation, as explained e.g. in Ref. ${ }^{[35}$. As discussed in Ref. ${ }^{36}$, however, some care must be paid to minimize the effect of normal modes thermostatting on the dynamical properties of interest.

Figure 2 shows that the use of an optimally-damped path integral Langevin thermostat ${ }^{35}$ does actually extend the stability of the method to outer timesteps well above the resonance barrier for the stretches. In fact, the deviations that are observed for the mean potential energy are due to the outer time step becoming inappropriate to integrate the long-range force (which is relatively quickly varying since we do not use any kind of range separation scheme). This additional stabilization is probably due to the coupling between ring-polymer modes and highfrequency centroid vibrations - which is also the cause for spectral broadening observed in thermostatted RPMD.

Figure 3 shows the velocity-velocity autocorrelation function for liquid water computed with TRPMD, and compare the results using full 32-beads PIMD and those obtained with RPC to 8 beads of the non-bonded interactions, and MTS to 1fs outer. Dynamical properties appear to be less strongly affected than thermodynamic averages when RPC/MTS techniques are pushed to their limits. Using RPC down to the centroid, or using a 3fs outer time step, lead to minor deviations from the reference. In summary, these benchmarks demonstrate the effectiveness of our implementation of combined RPC and MTS techniques in i-PI, and shows that with an appropriate thermostatting scheme one can push the stability limit of MTS schemes beyond the resonance barrier of RPMD, for the calculation of both static and dynamical properties (Fig. 2 and 3 ).

\section{NUCLEAR QUANTUM EFFECTS AND ELECTRONIC STRUCTURE METHODS: THE ZUNDEL CATION}

Having briefly reviewed the main ideas, and demonstrated the stability of RPC+MTS PIMD, let us move on to discuss a few aspects that are specific to a first principles context. Here, we simulate the quantum distributions of the Zundel cation at the MP2 level of theory, using semi-local DFT for RPC+MTS. While other choices are possible, this combination is really attractive, as it is general in nature, and the impact of the scheme can be very significant. Indeed, as system size increases, 


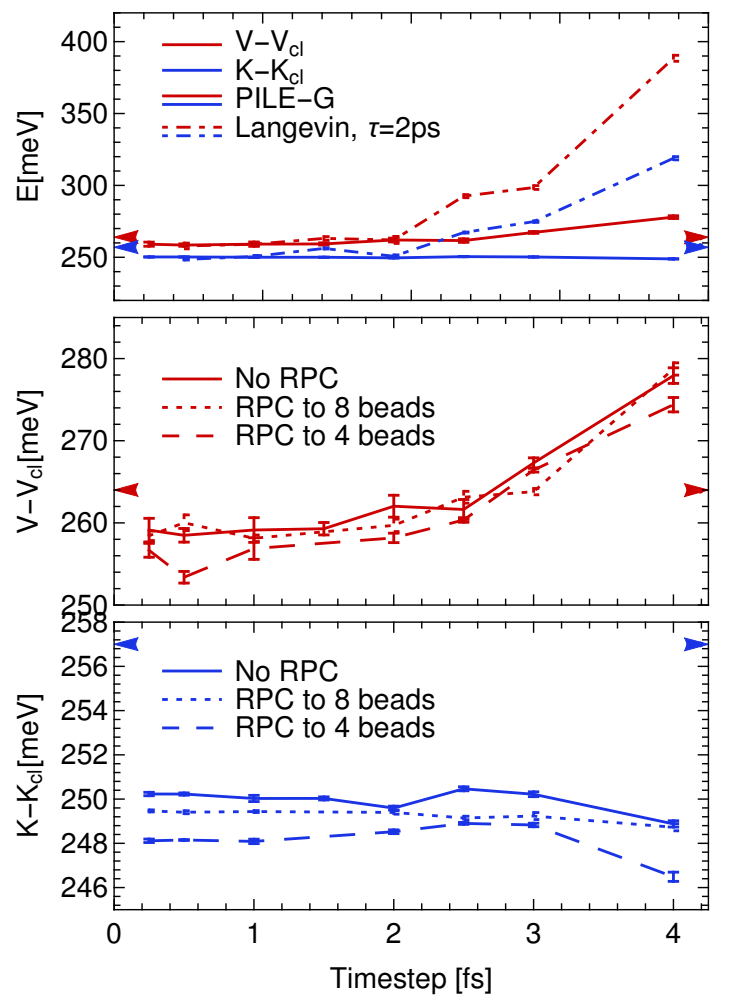

FIG. 2. Difference between quantum and classical expectation values of potential (top and middle) and kinetic (top and bottom) energy per molecule for a simulation of q-TIP4P/F liquid water at room temperature, as a function of the outer time step. The bonded terms were computed with a fixed inner time step of $0.25 \mathrm{fs}$, and propagated just outside the free ring-polymer part of the propagator. The non-bonded terms were computed less often, with a time interval as indicated on the horizontal axis. The blue and red curves correspond, respectively, to kinetic and potential energy. Solid curves correspond to 32 beads full PIMD simulation while dotted and dashed curves to simulations in which the nonbonded terms were contracted to 4 and 8 beads respectively. The dot dashed curves in the top panel correspond to simulations using a white noise Langevin thermostat with a time constant of 2 ps while the rest to simulations using a PILE-G thermostat.

MP2 and semi-local DFT display very different computational costs, since traditional implementations scale as $O\left(N^{5}\right)$ and $O\left(N^{3}\right)$ respectively. For example, three orders of magnitude time difference can be expected for samples of 64 water molecules ${ }^{2}$. The excellent scalability of our MP2 implementation $\frac{3738}{}$ nevertheless enables an acceptable time to solution. In the MP2+RPC context a large number of cheap DFT calculations must be combined with the expensive MP2 calculations, and the question of load balancing, avoiding idle processes as much as possible, imposes itself. As a simple, yet efficient, solution to this problem, we over-subscribe compute nodes using two processes per core. One process belongs to the $P$ DFT tasks, while one process belongs to the $P^{\prime}$ MP2

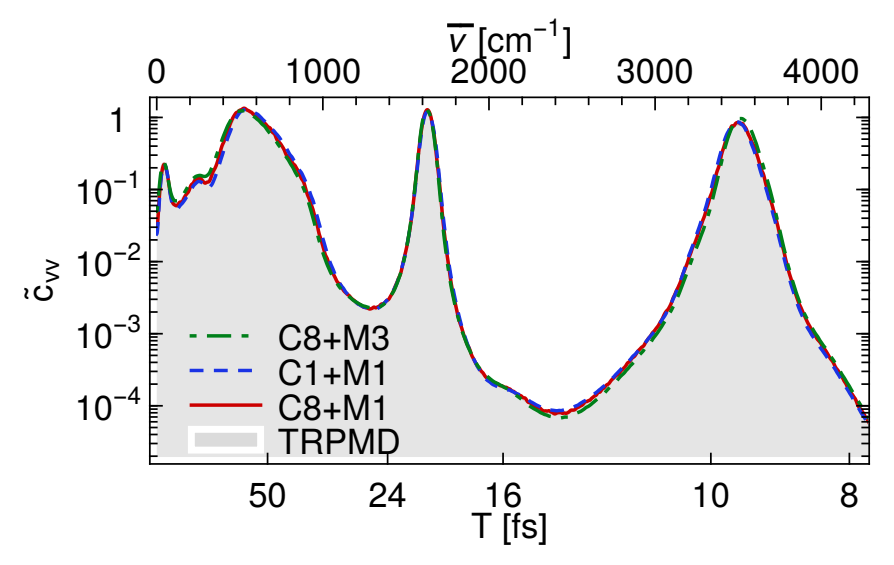

FIG. 3. The velocity-velocity autocorrelation function from $\mathrm{TRPMD}^{33}(\lambda=0.5)$ runs for $\mathrm{q}-\mathrm{TIP} 4 \mathrm{P} / \mathrm{F}$ water at $300 \mathrm{~K}$. A reference run (timestep $0.25 \mathrm{fs}, 32$ beads, shaded area) is compared with a run with 8-beads RPC for the non-bonded interactions, and an outer MTS timestep of $1 \mathrm{fs}(\mathrm{C} 8+\mathrm{M} 1$, red continuous line), with a run with 1-bead RPC and 1fs MTS (C1+M1, blue dashed line), and with a run with 8-beads RPC and 3fs MTS (C8+M3, green dot-dashed line).

tasks and the contracted DFT tasks, which run at the same time as MP2 39 . While the MP2 tasks computes, the full-path DFT tasks are effectively sleeping, and vice versa. In this way, idle resources are avoided, and only a small fraction of total wall time is spent in the DFT part, which is naturally parallel over the beads, while the computation for each bead is parallelised as well. If the contraction is pushed all the way to the centroid, the cost of performing MP2+DFT with MTS+RPC is basically the same as the cost of doing standard MP2 MD. An example of how these simulations can be set up in practice can be obtained from the authors.

The combination of MP2 with semi-local DFT employs the same computational setup as described in Ref. ${ }^{2}$. In particular, this approach is based on the resolution of identity Gaussian and Plane Waves (RI-GPW) which provides an efficient and scalable approach to perform MP2 based MD in gas and condensed phases. $37 / 38 / 41 / 42$ The Gaussian basis employed for MP2 is of the correlation consistent triple zeta quality $\underline{41}$ and is parametrized for the pseudopotentials employed $\stackrel{43}{43}$ The optimized density functional (ODF) discussed below is of the GGA family, starting from the PBE1W $W^{44}$ functional, for which the small basis and van der Waals D3 parameters ${ }^{45}$ have been specifically refitted in order to reproduce the energetics of bulk liquid water.2

Results for the mean kinetic energy of hydrogen and oxygen atoms, and the mean $\mathrm{O}-\mathrm{O}$ distance, for different simulations, are reported in Table [. The table caption summarizes the details of the simulations. The standard PBE functional underestimates the $\mathrm{H}$ kinetic energy with single-bead contraction, improving as expected with a contraction to two beads. This indicates that PBE differs too much from the reference, requiring a considerable 


\begin{tabular}{lccc}
\hline \hline Simulation & $K_{\mathrm{H}}[\mathrm{meV}]$ & $K_{\mathrm{O}}[\mathrm{meV}]$ & $d_{\mathrm{OO}}[\AA]$ \\
\hline MP2 & $148.4(5)$ & $58.9(4)$ & $2.413(4)$ \\
$\mathrm{PBE}$ & $143.1(5)$ & $57.5(4)$ & $2.445(4)$ \\
$\mathrm{PBE}+\mathrm{C}$ & $143.2(5)$ & $58.5(3)$ & $2.409(5)$ \\
$\mathrm{PBE}+\mathrm{C} 2+\mathrm{M}$ & $144.7(6)$ & $58.3(4)$ & $2.410(1)$ \\
$\mathrm{ODF}$ & $148.9(5)$ & $58.9(4)$ & $2.416(3)$ \\
$\mathrm{ODF}+\mathrm{C}$ & $149.3(5)$ & $59.6(4)$ & $2.413(4)$ \\
$\mathrm{ODF}+\mathrm{C}+\mathrm{M}$ & $149.7(3)$ & $59.3(3)$ & $2.410(3)$ \\
$\mathrm{ODF}+\mathrm{C}+\mathrm{M} 2$ & $149.4(4)$ & $59.4(3)$ & $2.412(5)$ \\
ODF+C+M+G & $148.0(9)$ & $59.4(5)$ & $2.412(2)$ \\
HFX+C+M & $155.3(7)$ & $60.6(5)$ & $2.411(3)$ \\
\hline \hline
\end{tabular}

TABLE I. Expectation values of quantum kinetic energies per $\mathrm{H}$ and $\mathrm{O}$ atom, and for the $\mathrm{O}-\mathrm{O}$ distance, in a simulation of a gas-phase Zundel cation at 300K. All simulations were performed using 32 beads and a base time step of $0.25 \mathrm{fs}$, except that using PIGLET $(+\mathrm{G})$ that used six replicas. PBE refers to use of the standard PBE generalized gradient approximation (GGA) functional ${ }^{40}$, ODF refers to a GGA optimized to $\mathrm{MP}^{2}$, and HFX to Hartree-Fock. Ring-polymer contraction was used to reduce the cost by computing the MP2 forces on the centroid only $(+\mathrm{C})$, or on two beads $(+\mathrm{C} 2)$. A multiple time step algorithm was also employed to evaluate such force only once every $1 \mathrm{fs}(+\mathrm{M})$ or $2 \mathrm{fs}(+\mathrm{M} 2)$. All simulations were run for $10 \mathrm{ps}$, including $1 \mathrm{ps}$ for equilibration. Statistical errors on the last digit are reported in parentheses.

increase in the number of MP2 replicas. Also HartreeFock is not very accurate, this time predicting a too large value of the $\mathrm{H}$ kinetic energy, despite the RPC correction. This suggests that the MTS strategy proposed in Ref ${ }^{[22}$, which combines HFX and MP2, cannot be used universally for RPC, at least not without a large number of correction beads. Hybrid functionals, which mix GGA exchange with Hartree-Fock exchange, will likely provide a suitable intermediate potential energy surface. However, as a computationally less demanding, but less universal alternative, an optimized semi-local DFT (ODF) shows excellent performance. The results reported in Table \are within the statistical error bars from the MP2 reference already without any RPC correction, and as a consequence it is not possible to detect statistically significant effects on these averages due to RPC and/or MTS. However, also in this case the RPC correction does have a noticeable effect. This is shown in Figure 4 using the joint probability distribution of the $\mathrm{O}-\mathrm{O}$ distance and the proton transfer coordinate. The difference between the MP2 and the $\mathrm{ODF}+\mathrm{C}$ is almost zero and smaller than ODF only. The remaining error is largely due to the statistical uncertainty in the probability distributions, which is relatively costly to reduce for the reference MP2 simulation that employs 32 MP2 beads.

Let us finally note that RPC and MTS methods can be seamlessly combined with other strategies to reduce the cost of a PIMD calculation: as demonstrated in Table 1 . the use of a colored-noise PIGLET thermostat ${ }^{11}$ makes it possible to reduce the number of baseline beads to six, that would be advantageous in cases where the cost of the GGA calculations is not negligible.

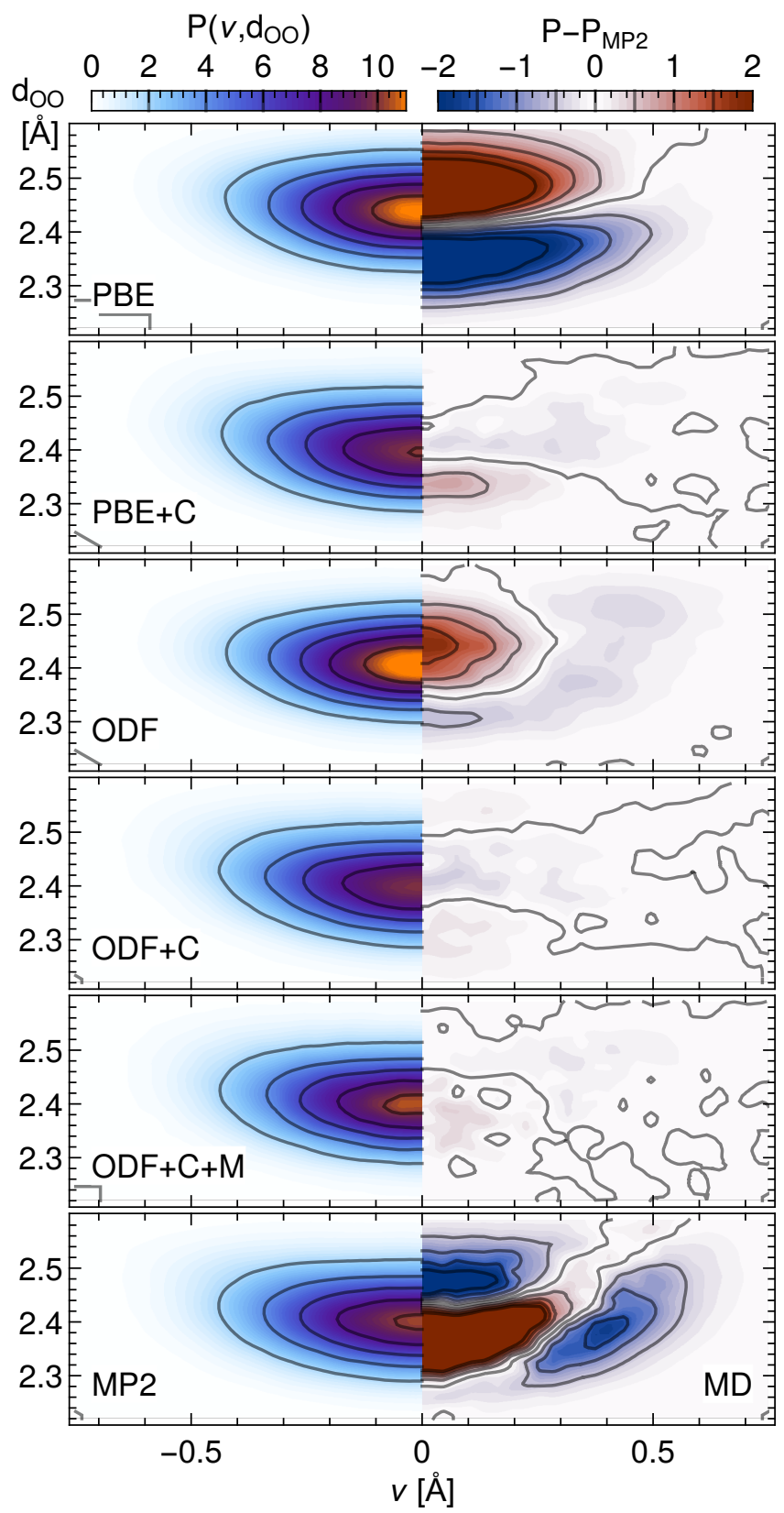

FIG. 4. Joint probability distribution for the proton transfer coordinate and the $\mathrm{O}-\mathrm{O}$ distance in the gas-phase Zundel cation at $300 \mathrm{~K}$, modeled with nuclear quantum effects and different electronic structure methods (left panels). Right-hand panels show the difference with respect to the MP2 reference. The bottom-right panel shows the difference between a classical MP2 MD simulation and the (quantum) MP2 reference.

A simple way to assess the viability of accelerating a simulation by means of RPC and/or MTS is to verify the magnitude and time scale for the variation of the correction potential. Fig. 5 shows the Fourier transform of the correction potential correlation function $\left\langle V^{\mathrm{S}}(t) V^{\mathrm{S}}(0)\right\rangle$. Clearly, the difference between MP2 and HFX contains strong high-frequency components, which would require 


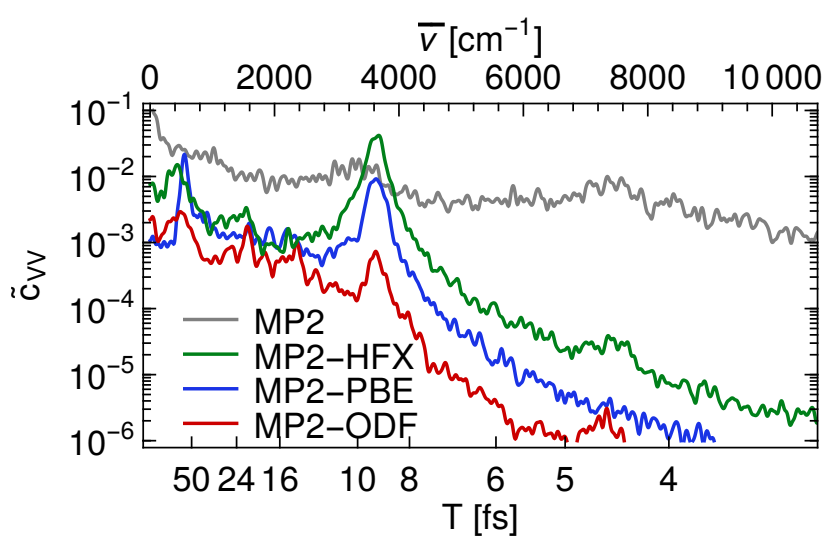

FIG. 5. Fourier-transform of the potential-potential correlation function for different RPC simulations. The curve for MP2 (in grey) is taken as a reference; being evaluated on 32 replicas, it also contains the high-frequency non-centroid modes of the ring polymer. The other two curves correspond to the correction potential (MP2 minus GGA) evaluated on the centroid for the HFX (green), PBE (blue) and optimized DFT (red) simulations. The horizontal scale indicates the period of different vibrational modes, for ease of reference. Since these simulations were heavily thermostatted for sampling and stability, the spectrum has no physical meaning, and is just a tool to assess the time scales that are relevant for different potential components.

a high number of beads in the contracted $V^{\mathrm{S}}$. Using the PBE GGA shows only a marginal advantage, as the spectrum still has a pronounced peak at the stretching frequencies. On the contrary, the ODF exhibits a smaller difference, and in particular a density of states smaller by an order of magnitude for the stretching region - which explains the excellent performance in reproducing quantum kinetic energies even without RPC.

\section{CONCLUSIONS}

Even on a small model system such as the gas-phase zundel cation a combination of ring-polymer contraction, multiple time step algorithms and a force-matching strategy to minimise the discrepancy between the expensive, post-HF calculation and a GGA DFT baseline makes it possible to model nuclear quantum effects at virtually no additional cost. Modular implementation in i-PI means that all sorts of similar schemes - combining different levels of theory, more or less converged basis sets, rangeseparated potentials, empirical or semi-empirical methods, etc. - could be tested and used without further coding effort ${ }^{46}$. Parallelism across replicas and potential components can be obtained by appropriately managing the execution on a HPC system, realizing a rudimentary but effective form of load balancing. Multiple time-stepping and ring-polymer contraction provide additional tools to reduce the computational expense for modeling quantum nuclei in ab initio simulations, that can be combined seamlessly with with high-order path integrals and correlated-noise techniques. Nuclear quantum effects can now be incorporated accurately and at low cost in simulations employing high level electronic structure theory. Given the importance of nuclear quantum effects and the generality of the approach, we expect this to become standard practice in the near future.

\section{ACKNOWLEDGEMENTS}

J.V. acknowledges financial support by the European Union FP7 in the form of an ERC Starting Grant under contract no. 277910. M.C and V.K acknowledge financial support by the Swiss National Science Foundation (project ID 200021-159896). This research was partly supported by NCCR MARVEL, funded by the Swiss National Science Foundation. Calculations were enabled by the Swiss National Supercomputer Centre (CSCS) under project ID ch5. During the preparation of this manuscript we became aware that $\mathrm{O}$. Marsalek and T. Markland were working on a related combination of DFT and self-consistent tight binding ${ }^{47}$ using MTS and RPC another excellent example of how inexpensive modelling of quantum nuclei can be achieved by coupling different levels of electronic structure theory.

${ }^{1}$ M. Del Ben, M. Schönherr, J. Hutter, and J. Vandevondele, J. Phys. Chem. Lett. 4, 3753 (2013).

${ }^{2}$ M. Del Ben, J. Hutter, and J. VandeVondele, J. Chem. Phys. 143, 054506 (2015).

${ }^{3}$ A. Zen, Y. Luo, G. Mazzola, L. Guidoni, and S. Sorella, J. Chem. Phys. 142, 144111 (2015).

${ }^{4}$ R. P. Feynman and A. R. Hibbs, Quantum Mechanics and Path Integrals (McGraw-Hill, New York, 1964).

${ }^{5}$ D. Chandler and P. G. Wolynes, J. Chem. Phys. 74, 4078 (1981).

${ }^{6}$ M. Parrinello and A. Rahman, J. Chem. Phys. 80, 860 (1984).

${ }^{7}$ S. S. Jang and G. A. Voth, J. Chem. Phys. 115, 7832 (2001).

${ }^{8}$ A. Pérez and M. E. Tuckerman, J. Chem. Phys. 135, 064104 (2011).

${ }^{9}$ M. Ceriotti, G. Bussi, and M. Parrinello, Phys. Rev. Lett. 103, 30603 (2009).

${ }^{10}$ M. Ceriotti, D. E. Manolopoulos, and M. Parrinello, J. Chem. Phys. 134, 84104 (2011).

${ }^{11}$ M. Ceriotti and D. E. Manolopoulos, Phys. Rev. Lett. 109, 100604 (2012).

${ }^{12}$ A. B. Poma and L. Delle Site, Phys. Rev. Lett. 104, 250201 (2010).

${ }^{13}$ T. E. Markland and D. E. Manolopoulos, J. Chem. Phys. 129, 024105 (2008).

${ }^{14}$ T. E. Markland and D. E. Manolopoulos, Chem. Phys. Lett. 464, 256 (2008).

${ }^{15}$ G. J. Martyna, M. E. Tuckerman, and M. L. Klein, J. Chem. Phys. 97, 2635 (1992).

${ }^{16}$ R. Zhou, E. Harder, H. Xu, and B. J. Berne, J. Chem. Phys. 115, 2348 (2001).

${ }^{17}$ M. J. Ferrarotti, S. Bottaro, A. Pérez-Villa, and G. Bussi, J. Chem. Theory Comput. 11, 139 (2015).

${ }^{18}$ M. Guidon, F. Schiffmann, J. Hutter, and J. Vandevondele, J. Chem. Phys. 128, 214104 (2008).

${ }^{19} \mathrm{~J}$. VandeVondele and U. Rothlisberger, The Journal of Chemical Physics 113, 4863 (2000).

${ }^{20}$ R. Iftimie, D. Salahub, D. Wei, and J. Schofield, The Journal of Chemical Physics 113, 4852 (2000). 
${ }^{21}$ M. J. McGrath, J. I. Siepmann, I.-F. W. Kuo, C. J. Mundy, J. VandeVondele, J. Hutter, F. Mohamed, and M. Krack, ChemPhysChem 6, 1894 (2005).

${ }^{22}$ R. P. Steele, J. Chem. Phys. 139, 011102 (2013).

${ }^{23}$ N. Luehr, T. E. Markland, and T. J. Martínez, J. Chem. Phys. 140, 084116 (2014).

${ }^{24}$ S. Fatehi and R. P. Steele, J. Chem. Theory Comput. 11, 884 (2015).

${ }^{25}$ H. Y. Geng, J. Comput. Phys. 283, 299 (2015).

${ }^{26}$ S. Habershon, T. E. Markland, and D. E. Manolopoulos, J. Chem. Phys. 131, 24501 (2009).

${ }^{27}$ M. Ceriotti, J. More, and D. E. Manolopoulos, Comput. Phys. Commun. 185, 1019 (2014).

${ }^{28} \mathrm{~J}$. VandeVondele, M. Krack, F. Mohamed, M. Parrinello, T. Chassaing, and J. Hutter, Comput. Phys. Commun. 167, 103 (2005).

${ }^{29}$ Q. Ma, J. A. Izaguirre, and R. D. Skeel, SIAM Journal on Scientific Computing 24, 1951 (2003).

${ }^{30}$ E. Barth and T. Schlick, The Journal of Chemical Physics 109, 1633 (1998).

${ }^{31}$ J. Cao and G. A. Voth, J. Chem. Phys. 101, 6168 (1994).

${ }^{32}$ I. R. Craig and D. E. Manolopoulos, J. Chem. Phys. 121, 3368 (2004).

${ }^{33}$ M. Rossi, M. Ceriotti, and D. E. Manolopoulos, J. Chem. Phys. 140, 234116 (2014).

${ }^{34}$ J. a. Morrone, T. E. Markland, M. Ceriotti, and B. J. Berne, J. Chem. Phys. 134, 14103 (2011).
${ }^{35}$ M. Ceriotti, M. Parrinello, T. E. Markland, and D. E. Manolopoulos, J. Chem. Phys. 133, 124104 (2010).

${ }^{36}$ M. Rossi, H. Liu, F. Paesani, J. Bowman, and M. Ceriotti, J. Chem. Phys. 141, 181101 (2014).

${ }^{37}$ M. Del Ben, J. Hutter, and J. VandeVondele, J. Chem. Theory Comput. 9, 2654 (2013).

${ }^{38}$ M. Del Ben, O. Schütt, T. Wentz, P. Messmer, J. Hutter, and J. VandeVondele, Comput. Phys. Commun. 187, 120 (2015).

${ }^{39}$ To achieve an effective load balancing, if $Q$ is the number of processes assigned to each of the $P$ DFT tasks, and $C_{\mathrm{MP} 2}$ and $C_{\mathrm{DFT}}$ are the scalar costs of each force evaluation at the two levels of theory, each of the contracted MP2 processes should be assigned about $P Q C_{\mathrm{MP} 2} /\left(m\left(C_{\mathrm{MP} 2}+C_{\mathrm{DFT}}\right)\right)$ processes, and $P Q C_{\mathrm{DFT}} /\left(m\left(C_{\mathrm{MP} 2}+C_{\mathrm{DFT}}\right)\right)$ processes should be given to each contracted DFT task.

${ }^{40}$ J. P. Perdew, K. Burke, and M. Ernzerhof, Phys. Rev. Lett. Phys. Rev. Lett. (USA), 77, 3865 (1996).

${ }^{41}$ M. Del Ben, J. Hutter, and J. Vandevondele, J. Chem. Theory Comput. 8, 4177 (2012).

${ }^{42} \mathrm{M}$. Del Ben, J. Hutter, and J. VandeVondele, J. Chem. Phys. 143, 102803 (2015).

${ }^{43}$ S. Goedecker, M. Teter, and J. Hutter, Phys. Rev. B 54, 1703 (1996)

${ }^{44}$ E. E. Dahlke, , and D. G. Truhlar*, The Journal of Physical Chemistry B 109, 15677 (2005).

${ }^{45} \mathrm{~S}$. Grimme, J. Antony, S. Ehrlich, and H. Krieg, The Journal of Chemical Physics 132, 154104 (2010).

${ }^{46}$ The development version we used here is provided in the SI. More recent versions can be obtained from the Authors.

${ }^{47} \mathrm{O}$. Marsakel and T. Markland, private communication. 